TITRE: RECHERCHE SUR LA SPIRITUALITÉ : LES DÉFIS DU RECRUTEMENT

Auteur(s): Bruno BÉLANGER ET Line BEAUREgARD

Publication: ReCRUtemEnT ET CONSENTEMENT À LA RECHERCHE : RÉALITÉS ET DÉFIS ÉTHIQUES

PAgES: $106-114$

ISBN: 978-2-7622-0357-8

Directeurs: Ana Marin, BéATrice Eysermann et Michel T. GIROUX

URI: HTTP://HDL.HANDLE.NET/11143/14115

DOI: HTTPS://DOI.ORG/10.17118/11143/14115 


\section{Recherche sur la spiritualité : les défis du recrutement}

Bruno Bélanger, agent de planification, de programmation et de recherche au Centre Spiritualitésanté de la Capitale-Nationale (CSsanté) du CHU de Québec - Université Laval.

Line Beauregard, agente de planification, de programmation et de recherche au Centre Spiritualitésanté de la Capitale-Nationale (CSsanté) du CHU de Québec - Université Laval. Membre de l’Ordre des psychologues du Québec.

Résumé : Au cours des dernières années, le CSsanté a réalisé des projets de recherche afin de mieux comprendre comment, dans l'environnement social pluraliste et laïque du Québec contemporain, se manifestait l'expression spirituelle de personnes en contexte de maladie. À partir de deux recherches réalisées auprès d'usagers en soins palliatifs, les auteurs font ressortir les défis rencontrés lors du recrutement des participants. Plusieurs de ces défis sont reliés au thème des recherches qui portaient sur les besoins spirituels et religieux des patients en fin de vie. II appert que l'utilisation des mots «spirituels» et «religieux» pouvait évoquer, tant pour les patients que pour les soignants, une recherche sur la foi telle que comprise dans la tradition judéo-chrétienne, ce qui a pu limiter la participation de personnes n'adhérant pas à ce courant. Afin d'obtenir des résultats plus représentatifs de la diversité des expériences spirituelles, il aurait été plus adéquat de présenter le projet comme une recherche portant sur le vécu intérieur en contexte de maladie.

Mots clés : spiritualité, soins palliatifs, recrutement, recherche, besoins spirituels

Summary: In the course of the last several years, the CSsanté produced several research projects so as to better understand how, within the pluralist and secular social environment of contemporary Québec, does spirituality manifest itself for persons within a context of illness. On the basis of two research studies conducted with clients in palliative care, the authors bring to the fore the various challenges which they encountered in their attempts to recruit participants. Several of these challenges were related to the research topics in question which dealt with the spiritual and religious needs of patients who were at the "end of life" phase of their lives. It would appear that the use of such words as "spiritual and religious" often evoked, for the patients as well as the caregivers, an inquiry into matters of faith such as is understood within a Judaeo-Christian tradition, which, it was found, often limited the participation of persons who did not adhere to this stream of consciousness. In an effort to obtain results which better represent the diversity of spiritual experiences in contemporary society, it would have been more appropriate, to present this project as a vehicle for research dealing with the inner lived experience of persons struggling with illness.

Keywords: spirituality, palliative care, recruitment, research, spiritual needs. 


\section{Pourquoi une recherche sur la spiritualité?}

Le Centre Spiritualitésanté de la Capitale-Nationale (CSsanté) s'investit depuis une douzaine d'années dans la recherche. La nécessité d'améliorer nos connaissances en nous appuyant notamment sur des recherches s'est accrue à mesure que se faisaient sentir, au sein du réseau de la santé, les conséquences des changements socioreligieux véhiculés par la Révolution tranquille. En effet, les professionnels impliqués à l'époque comme animateurs de pastorale ${ }^{2}$ prenaient progressivement conscience de transformations dans l'expression des besoins spirituels et religieux des patients et, par conséquent, de l'importance d'ajuster les modes d'intervention préconisés jusqu'à ce jour. Enfin, à l'époque des publications de plus en plus nombreuses, issues tout particulièrement de milieux anglophones, apparaissaient dans les champs situés à l'intersection de la spiritualité et de la santé.

C'est dans ce contexte que le CSsanté a souhaité mieux cerner comment, dans un environnement pluraliste et laïque, se manifestaient les grandes questions ou expressions de l'expérience spirituelle des patients. En d'autres mots, même si extérieurement de plus en plus de personnes n'adhéraient plus aux croyances véhiculées par une tradition spirituelle et une Église, elles continuaient de porter un ensemble de questions, de craintes, de sentiments reliés à leur vie intérieure. À travers ces recherches, nous espérions une meilleure compréhension de ce que vivent les patients en regard de leur vie spirituelle afin d'améliorer nos processus d’accompagnement dans la dernière étape de leur vie.

L'article qui suit mettra en lumière les principaux défis rencontrés lors du recrutement de patients dans le cadre de deux recherches qualitatives mises sur pied par le CSsanté entre 2007 et 2014 : une première étude concernant les patients suivis en soins palliatifs à domicile et une deuxième en soins palliatifs en milieu hospitalier. Ces recherches avaient deux objectifs spécifiques: 1) explorer et identifier les besoins spirituels et religieux des personnes en soins palliatifs; 2) élaborer une grille d'évaluation des besoins spirituels et religieux.

\section{Description du recrutement}

Concernant l'Étude portant sur les patients suivis à domicile, la collecte des données s'est effectuée sur le territoire du Centre de santé et de services sociaux (CSSS) de la Vieille-Capitale ${ }^{3}$. Trois équipes d'intervenants secteur Basse-Ville-Limoilou-Vanier, secteur Haute-Ville-des-Rivières, secteur Sainte-Foy-Sillery-Laurentien - ont été sollicitées pour proposer aux usagers de participer au projet de recherche.

Comme condition préalable à leur participation à l'étude, les personnes devaient être inscrites à un programme de soins palliatifs à domicile dispensé par un Centre local de services communautaires. Vingt-quatre entrevues ont ainsi été réalisées par deux interviewers sur une période de quatorze mois, soit de mai 2007 à juillet 2008.

1. Le Centre Spiritualitésanté de la Capitale-Nationale (CSsanté) a pour mission d'assurer la planification, la coordination, la prestation et l'évaluation d'activités cliniques de qualité afin de répondre aux besoins spirituels et religieux des personnes hospitalisées, hébergées ou suivies à domicile. Il regroupe tous les services de soins spirituels des établissements de santé et de services sociaux de la ville de Québec et de ses environs situés sur le territoire de la Capitale-Nationale. Composée de plus de 40 intervenants en soins spirituels, notre équipe de professionnels se déploie sur 35 sites répartis dans trois établissements du réseau de la santé et des services sociaux.

2. Au moment où nous avons entamé les recherches, le titre d'emploi des intervenants en soins spirituels était « animateur de pastorale».

3. Depuis l'entrée en vigueur de la projet de loi 10, le CSSS de la Vieille-Capitale est intégré au CIUSSS de la Capitale-Nationale. 
En tant que patients intégrés dans un programme de soins palliatifs, tous les participants avaient été informés par leur médecin, quelque temps avant leur participation à la recherche, qu'ils avaient une espérance de vie de moins de six mois, bien que certains aient pu ne pas avoir encore complètement assimilé cette information.

Pour la recherche auprès de patients en milieu hospitalier, les données ont été recueillies auprès de la clientèle en soins palliatifs uniquement à L'Hôtel-Dieu de Québec. Comme condition préalable à leur participation à l'étude, les personnes devaient être hospitalisées en soins palliatifs à L'Hôtel-Dieu de Québec. Entre juillet 2011 et avril 2013 (21 mois), 15 entrevues ont été réalisées au chevet des patients.

Pour les deux recherches, les intervenants ont présenté aux personnes sélectionnées un dépliant décrivant les objectifs et les modalités de leur participation au projet. Lorsqu'une personne acceptait de participer, l'intervenant transmettait son nom à un chercheur qui entrait rapidement en contact avec elle pour discuter plus en détail du projet et de ses implications et une rencontre était alors planifiée.

\section{Les participants}

Le tableau 1 présente la répartition des participants rencontrés à domicile selon leur groupe d'âge et selon leur sexe : seize femmes et huit hommes dont l'âge varie de 52 à 88 ans. Deux fois plus de femmes que d'hommes ont participé à la recherche et le groupe d'âge où l'on retrouve davantage de participants est celui des 69 ans et moins (Charboneau et Bélanger, 2013).

Tableau 1. Répartition des participants à domicile selon le genre et l'âge

\begin{tabular}{|l|l|l|l|}
\hline & Hommes & Femmes & Total \\
\hline 80 ans et plus & 2 & 4 & 6 \\
\hline 70 à 79 ans & 4 & 4 & 8 \\
\hline 69 ans et moins & 2 & 8 & 10 \\
\hline Total & 8 & 16 & 24 \\
\hline
\end{tabular}

Le tableau 2 présente la description des participants rencontrés en milieu hospitalier selon les mêmes critères que le tableau précédent. On remarque que le groupe d'âge où l'on retrouve davantage de participants est, comme à domicile, celui des 69 ans et moins; la répartition entre les hommes et les femmes est presque égale (Bélanger et Charbonneau, 2014).

Tableau 2. Répartition des participants en milieu hospitalier selon le genre et l'âge

\begin{tabular}{|l|l|l|l|}
\hline & Hommes & Femmes & Total \\
\hline 80 ans et plus & 1 & 1 & 2 \\
\hline 70 à 79 ans & 2 & 1 & 3 \\
\hline 69 ans et moins & 5 & 4 & 6 \\
\hline Total & 8 & 6 & 9 \\
\hline
\end{tabular}




\section{Les défis du recrutement}

Ces deux recherches ont posé des défis particuliers reliés au recrutement des participants. Nous les avons regroupés en six points : 1) élaborer le dépliant; 2) évoquer le mot «spiritualité»; 3) joindre les participants potentiels; 4) prendre rendez-vous; 5) amorcer l'entrevue; 6) distinguer les rôles de chercheur et d'intervenant.

\section{Défi un : Élaborer le dépliant}

Selon nous, la spiritualité ne réfère pas à une «chose» qu'on pourrait mettre à distance, mais avant tout à une posture d'écoute 4 . Selon cette manière d'envisager la spiritualité, nous voulions que les patients racontent, le plus librement possible, ce que l'expérience de la maladie et l'approche de la mort remuaient en eux. Ensuite, à partir de leur discours, nous souhaitions discerner leurs besoins spirituels et religieux. Nous avions opté pour un dépliant qui exprimait en quelques mots assez larges ce que nous cherchions à entendre : «Les questions qui vous préoccupent, vos ressources intérieures, l'importance de vos relations, vos craintes et vos joies.» Par ailleurs, et ce fut probablement une erreur, le dépliant indiquait aussi que la recherche était menée par notre service - à l'époque le Centre de pastorale de la santé et des services sociaux (CPSSS) $)^{5}$ et que l'objectif était de cerner les besoins spirituels et religieux des patients. Sans que nous le voulions, le document pouvait évoquer une démarche s'adressant particulièrement aux personnes ayant la foi ou adhérant ouvertement à une religion. Ainsi, malgré une description limpide de ce que nous attendions des patients, le choix d'inscrire le nom de l'organisation responsable et l'objectif final de la recherche a pu avoir comme conséquence de biaiser le recrutement.

\section{Défi deux : Évoquer le mot «spiritualité»}

Même si effectivement il était clair que ce que nous cherchions à entendre était de l'ordre de leur vie intérieure, de ce qu'ils vivaient, dès lors que les mots «spirituels» et «religieux» étaient imprimés sur notre dépliant, cette recherche prenait, dans la tête de plusieurs, une orientation d'abord religieuse. Également, même si l'accent était globalement placé sur la dimension spirituelle, il n’en demeure pas moins que des préjugés tenaces, au sein de la population en général, identifient encore étroitement spiritualité et religion. Notre recherche n'a pas échappé à cela! Plusieurs patients s'attendaient pratiquement à une rencontre avec un représentant d'une religion, et il n'était pas évident pour l'intervenant qui présentait le projet (prochain défi) de parler de la spiritualité sans référer à des concepts religieux. Conséquemment, il a fallu souvent repositionner, dès le début des entretiens, dans un langage très accessible, ce que nous entendions par «spiritualité» dans le cadre de cette recherche et ce que nous cherchions vraiment à saisir dans leur parcours de la maladie : non pas leur positionnement en regard d'un dieu ou d'une église, mais leur vie intérieure (ce qui pouvait bien sûr les amener à parler de Dieu ou de leur appartenance à une tradition religieuse, mais dans un second temps seulement).

\footnotetext{
4. Cette réflexion de Rosselet (2015) rend bien compte de cette réalité : « C'est d'ailleurs, selon mon expérience, l'une des caractéristiques de la spiritualité que de se vivre et se dire dans des aspects très concrets et simples de l'existence, mais qui ouvrent sur des hauteurs vertigineuses et des profondeurs abyssales! L'intervenant en soins spirituels, comme tout soignant d'ailleurs, sera attentif à écouter à l'arrière-fond des mots les plus simples les harmoniques spirituelles fondamentales qui les sous-tendent ; il entendra derrière les mots du quotidien le trouble et la quête spirituels qui s'y révèlent. » (p. 19).
}

5. Notre organisation a, suite à un nouveau cadre ministériel régissant la profession, changé de nom en 2011 pour devenir le Centre Spiritualitésanté de la Capitale-Nationale (CSsanté). Au moment où le dépliant a été élaboré, nous portions le nom de Centre de pastorale de la santé et des services sociaux (CPSSS). 


\section{Défi trois : Joindre les participants potentiels}

Puisque nous n'étions pas autorisés par notre CER à communiquer directement avec les participants potentiels pour les solliciter à participer au projet de recherche, nous avons dû passer par un intermédiaire. Le rôle de l'intermédiaire consistait à demander aux participants potentiels s'ils acceptaient qu'un membre de l'équipe de recherche entre en communication avec eux. Dans le cas des deux projets de recherche en question ici, ce sont des intervenants liés à l'équipe de soins qui ont fait ce premier contact. Il est devenu manifeste, après quelques entrevues, que les personnes qui avaient accepté de participer étaient presque toutes croyantes et pratiquantes, ce qui n'était pas représentatif de la diversité habituelle des patients rencontrés par les intervenants en soins spirituels (ISS). Était-ce le dépliant informatif qui biaisait le recrutement (comme nous l'avons évoqué plus haut)? Ou bien la manière dont il était présenté? Nous reconnaissons que le dépliant aurait dû être différent, mais émettons aussi l'hypothèse que la vision de l'intervenant qui réalisait ce premier contact a également joué un rôle de première importance. Si sa compréhension du thème de l'étude se résumait à une recherche sur la foi telle que comprise dans la tradition judéo-chrétienne, il est fort probable qu'il ait présenté cette recherche à des personnes qu'il savait ou pressentait être croyantes. Un intervenant avait d'ailleurs dit : «On pourrait demander à ce patient, je sais qu'il est croyant.» Il a donc fallu réexpliquer à quelques reprises aux intervenants le sens de notre recherche.

\section{Défi quatre : Prendre rendez-vous}

Après avoir obtenu l'accord d'un patient, l'intervenant transmettait au chercheur le nom, le numéro de téléphone et les autres informations de base (âge et diagnostic) le concernant. Le patient était joint par téléphone et nous fixions un moment pour la réalisation de l'entrevue. Cette étape a souvent constitué pour nous un autre défi. Malgré le fait que le patient ait donné son accord à l'intervenant, bien des événements pouvaient survenir entre les heures (ou les jours) qui séparent l'acquiescement verbal du patient et le téléphone du chercheur : maladie soudainement aggravée, humeur différente, visite imprévue, etc. Avec certains patients, le chercheur devait trouver le juste équilibre entre dénouer les craintes ou les appréhensions bien normales du patient d'une part, et, d'autre part, ne pas trop insister si le patient ne sou haitait plus se prêter à la recherche. Comme il est souvent difficile de recruter, le chercheur se devait d'être vraiment attentif à cette réalité afin de ne pas s'imposer. Il s'agissait ensuite de trouver le moment opportun pour la rencontre. En milieu hospitalier notamment, la dernière étape de vie se compte en nombre de jours. Par conséquent, la rencontre doit être très rapprochée, et il est essentiel de trouver le moment de la journée - ce dernier point est vite apparu fort important, car les temps libres peuvent être rares. En effet, ceci est déterminant puisque le chercheur doit s'assurer que le climat dans lequel l'entrevue se déroule est optimal, favorisant le partage de propos intimes et parfois très profonds.

\section{Défi cinq : Amorcer l'entrevue}

Nous avons constaté que l'étape du recrutement ne se termine pas forcément à la prise du rendez-vous, les débuts de l'entrevue font aussi partie du processus. En effet, les premières minutes de la rencontre, consacrées à la vérification de la bonne compréhension du patient en regard de sa participation et de la signature du feuillet de consentement, sont cruciales pour tout projet de recherche. Le nôtre n'a pas échappé à cette réalité. 
Voici un exemple significatif à cet égard. L'interviewer est entré chez une dame d'environ 70 ans manifestement communicative et qui, dès son arrivée, commence à partager un segment fort difficile de sa jeunesse. L'interviewer était déchiré entre le respect qu'il portait à ce qu'elle racontait avec beaucoup d'émotions (quelques larmes glissaient régulièrement sur ses joues) et son obligation de lui dire : «Je suis contraint, madame, de vous faire signer les formulaires de consentement et de vous informer à nouveau que je dois procéder à l'enregistrement de notre entretien». Dans ce cas, la signature a finalement pu être réalisée, mais il a été difficile de retrouver le rythme et la profondeur de la discussion de départ. Dans les premiers moments de la rencontre, l'interviewer avait même envisagé, pendant quelques minutes, de ne pas réaliser cette entrevue de recherche selon les critères éthiques convenus. Dans ce contexte aurait-il été éthiquement acceptable de laisser parler la personne, de l'enregistrer et, à la fin, de lui présenter le formulaire d'information et de consentement (FIC)? Cet exemple à notre sens illustre les défis qui peuvent toujours se présenter, même quand les principaux obstacles du recrutement semblent surmontés.

Ensuite, même après l'étape de la signature du FIC dûment terminée, il peut encore survenir une remise en question de la poursuite de l'entrevue. Par exemple, lors des premières questions, un autre participant avait évoqué discrètement une journée plus difficile et un manque d'énergie, sous-entendant qu'il n'était peut-être plus convenable de continuer l'entrevue. Il est vite apparu essentiel de porter attention à ces quelques mots indiquant un malaise et de signaler au patient, à nouveau, que nous n'attendions rien d'autre que sa parole à lui, qu'il n'y avait pas de bonnes ou de mauvaises réponses et qu'à tout moment il pouvait mettre fin à l'entretien. Il faut donc savoir entendre ce que le patient dit, même si c'est dit, quelquefois, indirectement. Parfois, simplement accepter de prendre le temps de bien écouter dénoue les craintes en regard de l'entrevue.

\section{Défi six : Distinguer les rôles de chercheur et d'intervenant}

Un dernier défi rencontré a souvent consisté à discerner si le patient avait bien saisi la différence entre le rôle de chercheur et celui d'intervenant en soins spirituels. Le simple mot «spiritualité», lors de la présentation du projet de recherche, suffisait, pour un certain nombre de patients, à dissocier notre approche d'une recherche ayant un caractère objectif et neutre. Bon nombre nous recevait comme si nous étions des ISS. Nous avons rapidement saisi qu'il était essentiel de présenter dans des mots simples et concrets ce qu'est un chercheur, ses objectifs, ses limites et ce que pourrait vivre avec eux un intervenant en soins spirituels dans une rencontre subséquente. Faire cette distinction est fondamental, puisque le contenu de l'entrevue peut s'avérer assez différent selon que le patient pense s'adresser à un chercheur ou à un ISS. De plus, ses attentes peuvent ne pas être satisfaites.

\section{Conclusion}

Enfin, un dernier aspect mérite d'être mentionné. L'entrevue, compte tenu du type de questions évoquées, pouvait mettre en lumière certaines souffrances chez la personne en regard de cette ultime étape de sa vie. Le chercheur pouvait constater, dans certains cas, qu'un patient gagnerait à être correctement accompagné par un professionnel des soins spirituels. Mais, pour différentes raisons (probablement des préjugés tenaces en regard de la religion que nous avons évoqués plus haut), certains participants ont manifesté clairement ne pas vouloir s'inscrire dans un tel processus. Bien que les participants aient exercé leur droit de refus, les laisser dans cet état de souffrance, suite aux questions d'entrevue, pouvait soulever pour le chercheur un certain malaise. 
Avec le recul, il est manifeste pour nous que, si nous avions à reprendre cette recherche, nous procéderions différemment en ce qui a trait au recrutement. Les termes «spiritualité» et «religion» ne seraient pas mentionnés dans notre dépliant, nos documents et nos présentations aux intervenants chargés du recrutement afin d'éviter les préconceptions liées à ces termes. II serait préférable de parler d'une recherche qui porte sur l'expérience intérieure dans le contexte de la maladie afin de rejoindre tous les patients, peu importent leurs croyances et leurs a priori face à la religion et la spiritualité. Nous serions ainsi en accord avec notre vision de la spiritualité et avec ce que nous voulons vraiment repérer : la prise de parole du patient sur ce qui se joue en lui, c'est-à-dire ses préoccupations, ses craintes, ses moments de bonheur, ses questions, etc. En fin de compte, les résultats de l'analyse du contenu des entrevues nous permettraient quand même de cerner leur expérience spirituelle. De plus, nos résultats seraient fort probablement plus représentatifs de la diversité des patients rencontrés dans le réseau de la santé et des services sociaux. 


\section{Bibliographie}

Bélanger, B. et Charbonneau, C. (2014). Reconnaître et accompagner l'expérience spirituelle en fin de vie. Les Cahiers francophones de soins palliatifs, 14 (1), 47-56.

Charbonneau, C. et Bélanger, B. (2013). Identification of spiritual and religious needs of terminally ill patients receiving palliative home care. Journal for the Study of Spirituality, 3 (1), 33-45.

Rosselet, F. (2015). La souffrance spirituelle | De l'enfermement à l'ouverture d'un espace. Spiritualitésanté, 8 (3), 18-21. 